Y-D Shen ${ }^{1,2}$

${ }^{1}$ Department of Ophthalmology, Changhua

Christian Hospital, Changhua, Taiwan

${ }^{2}$ Department of Ophthalmology, National Taiwan

University Hospital, Taipei, Taiwan

Correspondence: Y-D Shen,

Department of Ophthalmology,

National Taiwan University Hospital,

7, Chung-Shan S Road,

Taipei 100, Taiwan

Tel: + 886223123456 ext. 5205;

Fax: + 886223412875

E-mail: ydshen@ha.mc.ntu.edu.tw

Eye (2007) 21, 448-449. doi:10.1038/sj.eye.6702627;

published online 6 October 2006

Sir,

\section{Patient safety in cataract surgery}

We thank Dr Pandey and Mr Scott for their interest in our article. We support their observations drawing attention to the danger of incorrectly mounted Luer lock intraocular cannulae as a potential risk. Following notification of this case report, the National Reporting and Learning (NRLS) database at the National Patient Safety Agency (NPSA) was searched for any similar patient safety incidents. On the NRLS database, three more similar incidents and one near miss were retrieved. These cases along with the example reported by Pandey and Scott share a common consequential theme. This is of intraocular cannulae - such as Rycroft, hydro-dissection, or wound hydration cannulae - 'exploding' from syringes during cataract surgery. While root cause of events is not confirmed, failure to correctly engage Luer lock cannulae appears to be an issue. As such it might be best considered as 'user error' and is a patient safety incident, rather than a device failure. It is prudent to highlight device incidents to the Medicines and Healthcare products Regulatory Agency (MHRA) so that any device products concerned might be investigated further. We have now brought this matter to their attention.

We support the publicity of patent safety incidents so that the learning arising and any solutions emerging can be shared. Ophthalmic staff should be aware of this specific risk with mounting Luer lock syringes. We applaud the practice of Pandey and Scott of checking secure engagement of Luer lock mounted cannulae before their introduction into the eye. We commend the reporting of all patient safety incidents in both NHS and in independent sector care.

SP Kelly ${ }^{1}$ and NJ Astbury ${ }^{2}$

${ }^{1}$ Bolton Hospital NHS Trust, Lancashire, UK

${ }^{2}$ Norfolk and Norwich University Hospital NHS

Trust, Norwich, UK

Correspondence: SP Kelly, Bolton Hospital NHS Trust, Lancashire, UK

Tel: + 440120 4390694;

Fax: + 4401204390554

E-mail: spkelly@ntlworld.com

Eye (2007) 21, 449. doi:10.1038/sj.eye.6702626; published online 13 October 2006

Sir,

Locking the Luer lock

I read with interest the article on 'Patient safety in cataract surgery'. ${ }^{1}$ As stated in the article, penetration of the globe during periocular injections is rightly, a reportable incident. Complications however also occur with the use of syringes intracamerally. I would like to draw your attention to the case of an 85-year-old gentleman who underwent uneventful left phacoemulsification surgery. At the end of the procedure, a Luer lock syringe was used with a 27-gauge Rycroft cannula for stromal hydration around the corneal section. The cannula tip dislodged under pressure and penetrated the eye through the wound to cause a hyphaema, choroidal haemorrhage, and total retinal detachment.

He was managed initially with a vitrectomy, removal of the intraocular lens with drainage of the suprachoroidal haemorrhage, and silicone oil internal tamponade. He developed proliferative vitreoretinopathy with a perisilicone retinal detachment that was managed with a retinectomy and silicone oil top-up procedure 6 weeks later. The silicone oil was successfully removed 3 months later with secondary anterior chamber intraocular lens implantation; his final best-corrected visual acuity was $6 / 18$

Case reports of similar occurrences have been reported in the literature, one with the use of Luer locked syringe during hydrodissection ${ }^{2}$ and one with a nonthreaded syringe. ${ }^{3}$ 